\title{
Genome-wide analysis of fermentation and probiotic trait stability in Lactobacillus plantarum during continuous culture
}

\author{
Cuijiao Feng, ${ }^{1}$ Fuxin Zhang, ${ }^{1 *}$ Bini Wang, ${ }^{1}$ Liyuan Zhang, ${ }^{2}$ Yushan Dong,,${ }^{1}$ and Yuyu Shao ${ }^{1 *}$ (1) \\ ${ }^{1}$ College of Food Engineering and Nutritional Science, Shaanxi Normal University, Xi'an 710119, China \\ ${ }^{2}$ School of Biotechnology, Jiangnan University, Wuxi 214122, China
}

\begin{abstract}
Trait stability of Lactobacillus plantarum was studied following daily subculture over a 90-d period. Acid and bile tolerance, self-aggregation ability, cell hydrophobicity, pathogen inhibition activity, and cholesterol removal ability of cultures subcultured 30 (Lp30), 60 (Lp60), or 90 (Lp90) times were not significantly different from the original strain (Lp0). However, carbohydrate metabolism patterns did change; the Lp0 culture was unable to use D-sorbitol, $\alpha$-methyl-D-mannose, and D-raffinose, whereas Lp30, Lp60, and Lp90 cultures could. Furthermore, gluconate and gentiobiose were fully used by the Lp0 culture but only poorly used by the Lp30, Lp60, and Lp90 cultures. Milk fermentation test confirmed that $L$. plantarum was unable to use lactose throughout laboratory evolution. Six non-synonymous mutations in genome of the Lp30, Lp60, and Lp90 cultures were identified by whole-genome sequencing, including mutant gene encoding the phosphoglycerate mutase, which is closely related to the transport and metabolism of carbohydrates. These mutations may play an important role in changes of carbohydrate metabolism patterns observed. Understanding the evolutionary characteristics of $L$. plantarum will help in development for food industry.
\end{abstract}

Key words: fermentation property, probiotic trait, laboratory evolution, stability, whole-genome sequencing

\section{INTRODUCTION}

Probiotics are live microorganisms that are beneficial to host when consumed in adequate amounts (FAO and

Received June 1, 2019.

Accepted September 11, 2019.

*Corresponding authors: fuxinzh@snnu.edu.cn and yuyushao@ outlook.com
WHO, 2006). For use as supplements, probiotics must be successfully produced on an industrial scale by longterm or continuous fermentation; during this process they must retain the genetic stability and physiological attributes that make them as the effective probiotics (Tuomola et al., 2001). Legislation states that probiotics can only be used in infant foods if it has been proved that they are genetically stable (Agostoni et al., 2004). Acid and bile tolerance capabilities are essential attributes of probiotics that ensure they remain viable following ingestion and reach their site of action in the intestinal tract (Dunne et al., 2001). Adhesion is considered to be an ideal probiotic feature since it promotes intestinal retention of probiotics and their interaction with host epithelial and immune cells (He et al., 2017). The probiotic with antibacterial activity can inhibit the growth of pathogenic bacteria in the intestinal tract, thereby maintaining the intestinal flora balance (NikuPaavola et al., 1999). Meantime, some probiotics with cholesterol-lowering function are of great significance in reducing the incidence of cardiovascular disease (Guo and Zhang, 2010). Some species have unique enzyme systems that participate in decomposition of carbohydrates (Kerr, 2004). Some probiotics can also use lactose to produce lactic acid in milk fermentation and thus are widely used as a starter culture in making cheese (Milesi et al., 2008) and yogurt (Manalis and Magkanari, 2006). The stability of these characteristics during long-term production should be considered and evaluated before their application.

Controlled laboratory evolution studies are useful for studying genetic changes in bacteria and the influence of these changes on phenotypic traits during long-term culture or repeated subculture (Buckling et al., 2009). Whole-genome sequencing technology enables the study of gene mutations in bacteria brought about by evolution in a given environment (Bryant et al., 2012). The stability of the probiotics such as Lactobacillus casei (Morelli and Campominosi, 2002), Lactobacillus rhamnosus (Elo et al., 1991), and Lactococcus lactis (Bachmann et al., 2012) were reported. Lactobacillus 
plantarum ATCC 14917 is a probiotic that is a reference strain widely used in scientific research (TomaroDuchesneau et al., 2014; Wang et al., 2016). In this study, the stability of L. plantarum ATCC 14917 was evaluated. The fermentation and probiotic characteristics (i.e., phenotypic stability) of L. plantarum ATCC 14917 strain were determined after daily subculture for $30 \mathrm{~d}$ (Lp30), $60 \mathrm{~d}$ (Lp60), and $90 \mathrm{~d}$ (Lp90). Further analysis of the genetic stability of lineages at the wholegenome level was made to help identify the mechanisms for any phenotypic changes and provide the information necessary for development in food industry.

\section{MATERIALS AND METHODS}

\section{Continuous Subculture of L. plantarum}

The original Lactobacillus plantarum strain ATCC 14917 was obtained from the American Type Culture Collection; it was activated in de Man, Rogosa, and Sharpe (MRS) broth and streaked onto agar plates. After incubation at $28^{\circ} \mathrm{C}$ for $48 \mathrm{~h}, 2$ individual colonies were selected randomly and each inoculated into MRS broth. After $24 \mathrm{~h}$ of incubation at $28^{\circ} \mathrm{C}$, the 2 strains were designated as the 0 -generation ( $\mathbf{L p 0}$; i.e., the population present before continuous subculture began). The $2 \mathrm{Lp} 0$ cultures were then subcultured, every $24 \mathrm{~h}$ for $90 \mathrm{~d}$, into fresh lactic acid bacteria susceptibility test medium broth consisting of 10\% MRS and $90 \%$ ISO-Sensitest broth at a rate of $1 \%$ (vol/vol) inoculum, and incubated at $28^{\circ} \mathrm{C}$ for continuous subculture (Dong et al., 2019).

\section{Carbohydrate Metabolism Patterns of L. plantarum}

The carbohydrate metabolism patterns of L. plantarum sampled from Lp0, Lp30, Lp60, and Lp90 were analyzed using the API 50 CHL kit (bioMérieux, France). The API $50 \mathrm{CHL}$ is a medium consisting of 49 fermentable carbohydrate API $50 \mathrm{CH}$ test strips. The inoculated test strip produces acid under anaerobic conditions and the $\mathrm{pH}$ change causes the color of the medium indicator to change, indicating whether the $L$. plantarum could ferment the corresponding carbohydrate.

The ability of lactose utilization in L. plantarum was verified by milk fermentation test. The samples from Lp0, Lp30, Lp60, and Lp90 were inoculated into sterilized skim milk at a rate of $1 \%(\mathrm{vol} / \mathrm{vol})$ inoculum and fermentation allowed to proceed at $37^{\circ} \mathrm{C}$. Titratable acidity was measured every $1 \mathrm{~h}$ following the methods of sodium hydroxide titration (AOAC International, 1999). The $\mathrm{pH}$ was determined using a precision $\mathrm{pH}$ meter at the same time. Measurements from each sample were performed in triplicate.

\section{Acid Tolerance of L. plantarum}

Acid tolerance was evaluated following the method of Hyronimus et al. (2000) with some modifications. The Lp0, Lp30, Lp60, and Lp90 cultures were inoculated into fresh MRS medium at either $\mathrm{pH} 2$ or $\mathrm{pH} 3$, and incubated for $3 \mathrm{~h}$ at $37^{\circ} \mathrm{C}$ and then diluted 10 times with PBS buffer (0.1 $M, \mathrm{pH} 7.2)$. The original and residual viable counts were determined by plate counting in serial dilutions with sterilized saline. The measurements were determined in triplicate.

\section{Bile Tolerance of L. plantarum}

Bile tolerance was determined following the methods of Tuomola et al. (2001) and Walker and Gilliland (1993), with some modifications. The Lp0, Lp30, Lp60, and Lp90 cultures were inoculated into MRS broth containing $0.1 \%$ oxgall at a rate of $1 \%$ (vol $/ \mathrm{vol}$ ) inoculum to broth, and incubated at $37^{\circ} \mathrm{C}$ in a water bath. The MRS broth without oxgall was used as a control. Optical density (OD) was measured at $600 \mathrm{~nm}$ per hour until the OD increased by 0.3 units and the time required was recorded. The difference between the time required for the absorbance of the Lp0, Lp30, Lp60, and Lp90 cultures with or without oxgall to increase by 0.3 units was the lag time (LT). Bile tolerance capability of the cultures was based on the length of the LT. Measurements for each strain were done in triplicate.

\section{Adhesion Ability of L. plantarum}

Cell hydrophobicity and self-aggregation capability are used to measure the adhesion characteristics of probiotics. The Lp0, Lp30, Lp60, and Lp90 cultures were centrifuged at $2,500 \times g$ for $7 \mathrm{~min}$, and the precipitates were washed twice with PBS buffer $(0.1 \mathrm{M}, \mathrm{pH} 7.2)$. The absorbance (OD at $600 \mathrm{~nm}$ ) was adjusted to $0.25 \pm$ 0.05 , and then the cultures were dispensed into the test tubes and allowed to stand at $37^{\circ} \mathrm{C}$. The absorbance was measured at 0-, 2-, 16-, 20-, and 24-h intervals (Collado et al., 2008). The self-aggregation rate $(A)$ was calculated according to the following formula:

$$
A(\%)=\left(1-\frac{A_{t}}{A_{0}}\right) \times 100,
$$

where $A_{0}$ is the absorbance value at $0 \mathrm{~h}$, and $A_{t}$ is the absorbance value at time interval $t$. 
Cell hydrophobicity of Lp0, Lp30, Lp60, and Lp90 cultures was determined based on the methods of Patel et al. (2009). The Lp0, Lp30, Lp60, and Lp90 cultures were centrifuged at $9,400 \times g$ at $4^{\circ} \mathrm{C}$ for $3 \mathrm{~min}$, and washed twice with PBS buffer. The precipitates were resuspended in PBS buffer and the absorbance measured at $600 \mathrm{~nm}$ as $A_{0}$. Then each bacterial suspension was mixed with an equal volume of xylene (nonpolar solvent), chloroform (acid solvent), and ethyl acetate (basic solvent), vortexed for $5 \mathrm{~min}$, and allowed to stand for 30 min until the mixture had separated into 2 phases. The absorbance of the water phase measured at $600 \mathrm{~nm}$ as $A_{1}$. Cell hydrophobicity was calculated according to the following formula:

$$
\text { Cell hydrophobicity }(\%)=\left(1-\frac{A_{1}}{A_{0}}\right) \times 100 \text {. }
$$

\section{Pathogen Inhibition Activity of L. plantarum}

The pathogen inhibition activity of $L$. plantarum ATCC 14917 was analyzed using the agar diffusion method (Lin et al., 2007). Pathogenic Escherichia coli and Staphylococcus aureus were used as the indicator strain in the test. These indicator strains were cultured overnight $\left(37^{\circ} \mathrm{C}\right)$ in brain heart infusion broth and diluted to 0.5 McFarland with sterile PBS ( $\mathrm{pH} 7.2$ ), and then the $0.1 \mathrm{~mL}$ of cell dilution solution were spread on the nutrient agar. The L. plantarum culture solution after $24 \mathrm{~h}$ cultivation was centrifuged to obtain a supernatant $(12,000 \times g, 10 \mathrm{~min})$, and a sterilized $\left(121^{\circ} \mathrm{C}\right.$, $20 \mathrm{~min}$ ) 6-mm diameter circular filter paper piece was immersed in the supernatant for $2 \mathrm{~h}$. The paper was then applied to an agar plate coated with indicator bacteria, followed by overnight incubation at $37^{\circ} \mathrm{C}$. The diameter of the inhibition zone on the agar plate was measured. The pathogen inhibition activities were classified as none $(-)$, weak $(+)$, middle $(++)$, and strong $(+++)$, according to the diameters of inhibition zone of $<5,>5,>10$, and $>15 \mathrm{~mm}$, respectively. Each assay was performed in triplicate.

\section{Cholesterol Removal Ability of L. plantarum}

Samples were prepared according to the methods described by Gilliland et al. (1985), then $0.1 \mathrm{~g}$ of cholesterol, $0.2 \mathrm{~g}$ of oxgall, $0.1 \mathrm{~g}$ of sucrose esters, $1 \mathrm{~mL}$ of Tween-80, and $3 \mathrm{~mL}$ of glacial acetic acid were mixed in $60^{\circ} \mathrm{C}$ water bath and treated with ultrasound for $15 \mathrm{~min}$ at $300 \mathrm{~W}$. The mixture was then added to MRS broth to obtain MRS-CHOL medium containing $0.1 \mathrm{mg} /$
$\mathrm{mL}$ cholesterol. The MRS-CHOL was sterilized after adjusting the $\mathrm{pH}$ between 6.0 and 7.0. The fresh MRS broth culture of L. plantarum was inoculated $(2 \%)$ into MRS-CHOL and incubated at $37^{\circ} \mathrm{C}$ for $24 \mathrm{~h}$, and then the supernatant was obtained by centrifugation for 20 min at $10,000 \times g$ at $4^{\circ} \mathrm{C}$. The cholesterol content in supernatant was determined by the ammonium ferric sulfate method (Lu et al., 2015). The cholesterol removal amount was estimated by subtracting the cholesterol amount of the used broth from the control broth. Each sample was performed in triplicate.

\section{Whole-Genome Sequencing of L. plantarum and Bioinformatics Analysis}

Genomic DNA from Lp0, Lp30, Lp60, and Lp90 cultures was extracted using the Qiagen kit (Qiagen, Hilden, Germany). The concentration and purity of extracted DNA were tested and whole-genome sequencing was done using the Illumina HiSeq 2,500 system (Illumina Inc., San Diego, CA). The sequencing data, in FASTA format, were analyzed by CLC genomics workbench software (v9.5.2; Qiagen Inc.) including quality control, trimming, mapping reads to references, removing duplicate reads, and detecting mutants. The match score, mismatch score, length fraction, and similarity fraction were $1,2,0.5$, and 0.8 , respectively. For duplication removal, a minority sequence of 20 was used. The mutations were detected under the minimum count of 2 and minimum frequency of $1 \%$. Gene stability of L. plantarum during continuous subculture was evaluated by analyzing the gene mutations in the Lp30, Lp60, and Lp90 cultures and comparing them with the gene mutations in the Lp0 culture.

\section{Statistical Analyses}

One-way ANOVA was done using SPSS Statistics 22.0 software (IBM Corp., Armonk, NY) to identify statistically significant differences in the experimental data at the $5 \%$ level $(P<0.05)$. Figures were plotted using Origin (v8.5) software (OriginLab Corporation, Northampton, MA).

\section{Accession Number}

The raw data of 8 sequencing samples (2 samples for each of Lp0, Lp30, Lp60, and Lp90 cultures) in the FASTQ format were obtained using the Illumina HiSeq PE150 (paired-end 150-bp sequencing) sequencing platform. All these sequences were deposited in the NCBI Sequence Read Archive (SRA) database (https:/ 
/www.ncbi.nlm.nih.gov/sra) and the accession number is PRJNA558098.

\section{RESULTS}

\section{Carbohydrate Metabolism Patterns of L. plantarum During Continuous Subculture}

Metabolic capabilities of the Lp0, Lp30, Lp60, and Lp90 cultures were evaluated for 49 carbohydrates (Table 1), showing that the Lp0 culture metabolized a variety of carbohydrates including ribose, galactose, mannose, gentiobiose, and gluconate, but was unable to metabolize other carbohydrates including D-sorbitol,

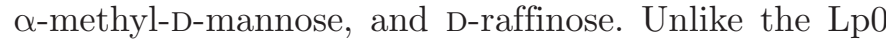
culture, the Lp30, Lp60, and Lp90 cultures were able to metabolize D-sorbitol, $\alpha$-methyl-D-mannose, and Draffinose. Furthermore, whereas the Lp0 culture could fully metabolize gluconate and gentiobiose, the Lp30, Lp60, and Lp90 cultures could only weakly metabolize these compounds. Acid production by Lp0, Lp30, Lp60, and Lp90 cultures after 6 h of milk fermentation increased, but no significant differences $(P>0.05)$ were observed in acidity or $\mathrm{pH}$ among the Lp0, Lp30, Lp60, and Lp90 cultures (Table 2).

\section{Acid and Bile Tolerance of L. plantarum During Continuous Subculture}

Viable counts for the Lp0, Lp30, Lp60, and Lp90 cultures were $10^{9}$ to $10^{10} \mathrm{cfu} / \mathrm{mL}$, which decreased to $10^{6}$ to $10^{8} \mathrm{cfu} / \mathrm{mL}$ after $3 \mathrm{~h}$ incubation in a low $\mathrm{pH}$ environment, and no significant difference $(P>0.05)$ was observed in viable counts for Lp0, Lp30, Lp60, and Lp90 cultures after acid treatment (Figure 1). No significant difference $(P>0.05)$ was observed in bile tolerance for Lp0, Lp30, Lp60, and Lp90 in $0.1 \%$ oxgall during continuous subculture; the LT varied between 2.73 and $2.89 \mathrm{~h}$ (Table 3 ).

\section{Adhesion Ability of L. plantarum During Continuous Subculture}

No significant difference $(P>0.05)$ was observed in the cell hydrophobicity of Lp0, Lp30, Lp60, and Lp90 cultures (Table 4). The cell hydrophobicity of the 3 cultures as determined with chloroform and xylene $(>90 \%)$ was higher than when determined using ethyl acetate $(<80 \%)$. During the 24 -h culture period, the self-aggregation rate of Lp0, Lp30, Lp60, and Lp90 increased to 59.87, 59.66, 60.01, and 58.49, respectively, but these values were not significantly different $(P>$ 0.05) from each other.

\section{Pathogen Inhibition Activity of L. plantarum During Continuous Subculture}

The fermentation supernatant of L. plantarum had an inhibitory effect on the growth of $S$. aureus and $E$. coli, especially the inhibition of $S$. aureus was stronger than that on $E$. coli inhibition zone diameter (Table $5)$. The pathogen inhibition activity of $L$. plantarum remained stable during continuous subculture.

\section{Cholesterol Removal Ability of L. plantarum During Continuous Subculture}

The distribution of cholesterol removal rate for $L$. plantarum fermentation supernatant is shown in Figure 2. All strains had cholesterol removal ability with the average removal rate of $28.92 \%$, and no significant difference was observed in the cholesterol removal rate among Lp0, Lp30, Lp60, and Lp90 cultures.

\section{Detection of Gene Mutations}

Mapping of the tested sequences to the reference sequence of Lactobacillus plantarum ATCC 14917 is presented in Table 6, showing that the matching rate of the sequences of all samples was higher than $98 \%$. The quality distribution of sequencing data is presented in Figure 3 showing high-quality sequences were obtained, which could be used for further analysis. Six non-synonymous mutations related to phenotypic characteristics were detected (Table 7 ), including 1 single nucleotide variant and 1 insertion in 2 genes encoding hypothetical protein and bile acid $7-\alpha$ dehydratase, respectively, and 4 deletions in genes regulating sugar transport protein, YibE/F-like protein, phosphoglycerate mutase 1 family, and putative foldase protein PrsA 2. The information of matched sequence, mutation site, and reference gene sequence were established as a track list by using CLC Genomics Workbench to locate the mutations. The gene encoding YibE/F-like protein had deletion of 4 bases at the position of $337,385-337,388$, which is presented in Figure 4.

\section{DISCUSSION}

Although probiotics are mainly taken for their beneficial health effects, they are widely found in fermented products, which are more palatable for consumers (Parvez et al., 2006). In the present study, the carbohydrate metabolism analysis showed that lactose could not be used by L. plantarum strain ATCC 14917. Milk fermentation test verified the low-level lactic acid production in this strain, indicating it is not suitable 
Feng et al.: STABILITY OF LACTOBACILLUS PLANTARUM

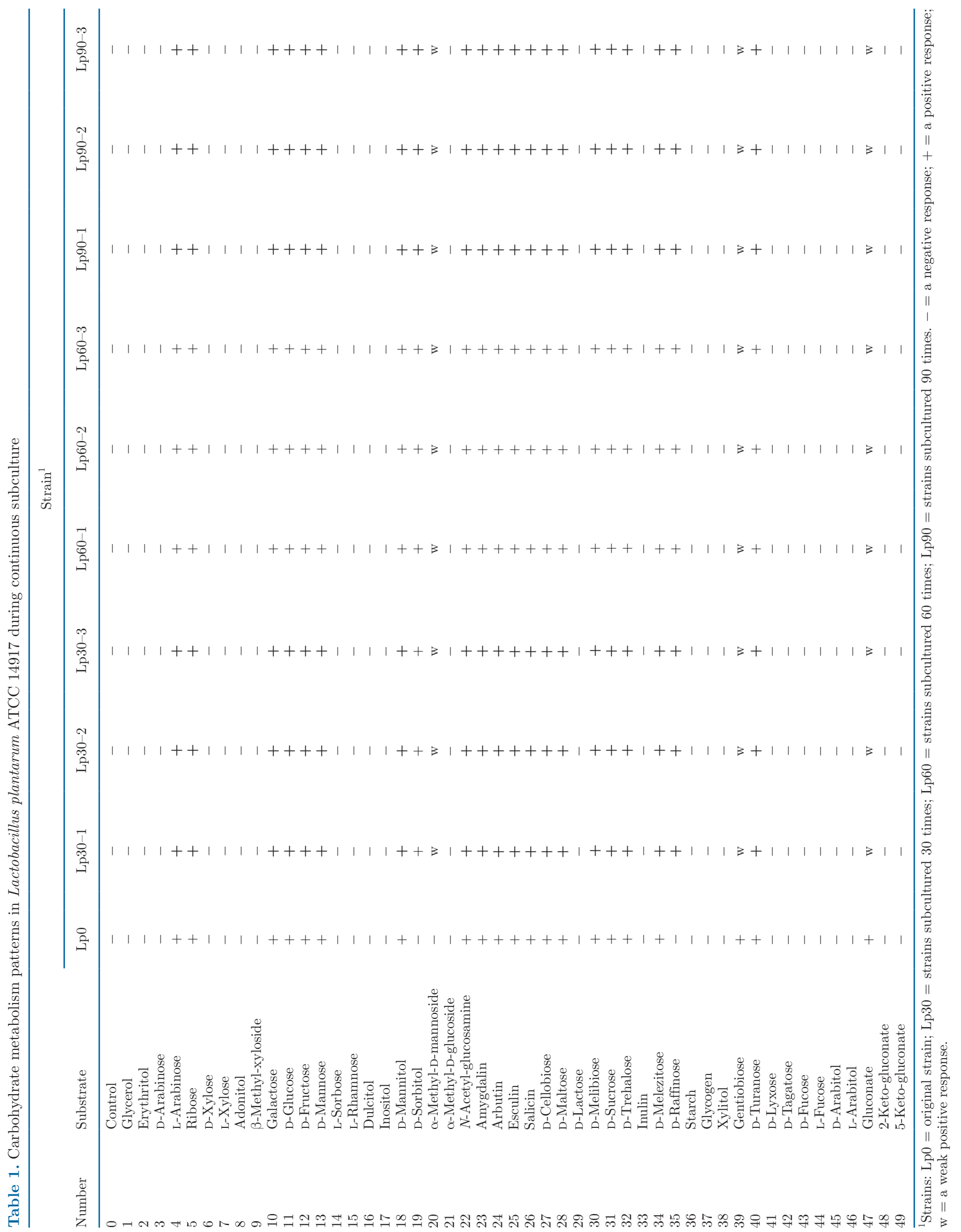




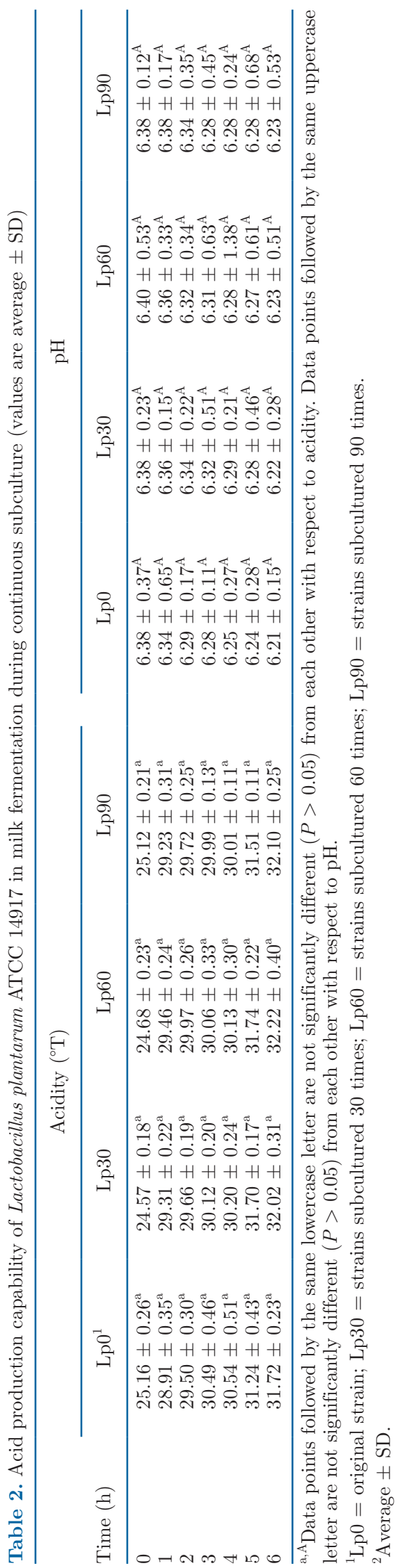

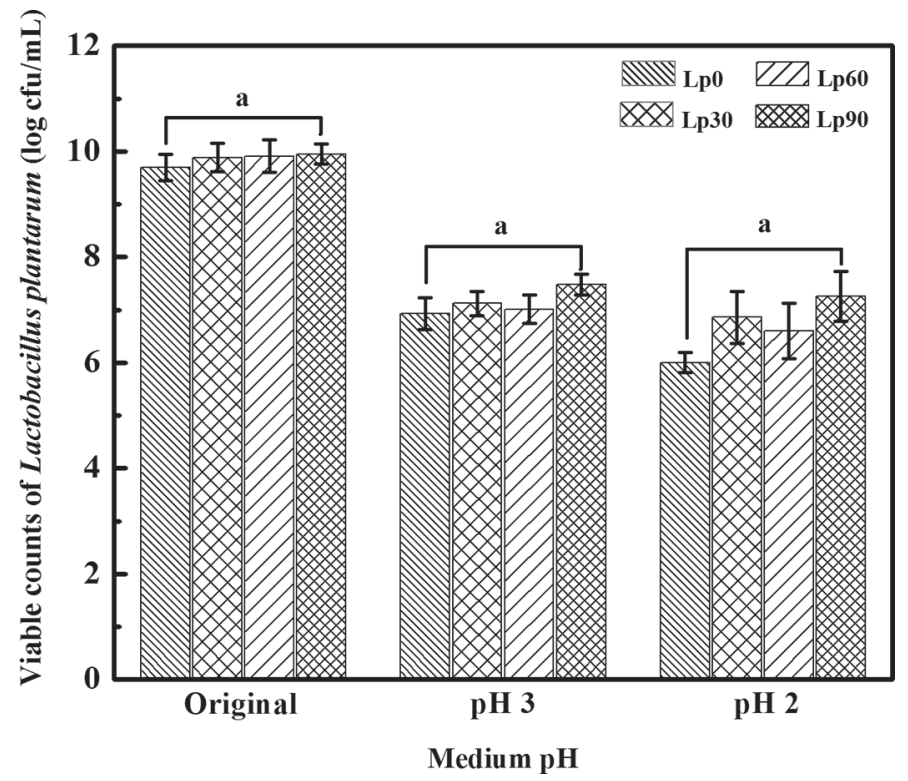

Figure 1. Acid tolerance of Lactobacillus plantarum ATCC 14917 during continuous subculture. $\mathrm{x}$-Axis: different $\mathrm{pH}$ conditions in the culture medium of Lactobacillus plantarum; y-axis: viable counts of Lactobacillus plantarum $(\log \mathrm{cfu} / \mathrm{mL}) . \mathrm{Lp} 0=$ original strain; $\mathrm{Lp} 30=$ strains subcultured 30 times; Lp60 = strains subcultured 60 times; Lp90 = strains subcultured 90 times. Error bars indicate SD. The identical lowercase letters indicate that no significant differences were observed in viable counts among strains at different $\mathrm{pH}$ conditions.

for milk fermentation. Medina et al. (2001) and NietoArribas et al. (2009) evaluated L. plantarum strains isolated from cheese and also found weak acid production properties or a slow acid production rate. According to bioinformatics analysis, the whole-genome sequence of $L$. plantarum ATCC 14917 contained galRKTEM, indicating that it can use galactose, which is consistent with the results presented in galactose me-

Table 3. Bile tolerance of Lactobacillus plantarum ATCC 14917 during continuous subculture (values are average $\pm \mathrm{SD}$ )

\begin{tabular}{lccc}
\hline & \multicolumn{3}{c}{$\begin{array}{c}\text { Time required for } \mathrm{OD}_{600} \\
\text { increasing by } 0.3 \operatorname{unit}^{2}(\mathrm{~h})\end{array}$} \\
\cline { 2 - 3 } & \multicolumn{3}{c}{$\begin{array}{c}\text { MRS broth } \\
\text { Strain }\end{array}$} \\
\cline { 2 - 3 } & MRS $^{4}$ broth & $6.1 \%$ oxgall & LT $^{3}(\mathrm{~h})$ \\
\hline Lp0 & $3.75 \pm 0.21^{\mathrm{a}}$ & $6.48 \pm 0.37^{\mathrm{a}}$ & $2.73 \pm 0.16^{\mathrm{a}}$ \\
Lp30 & $3.70 \pm 0.10^{\mathrm{a}}$ & $6.50 \pm 0.25^{\mathrm{a}}$ & $2.80 \pm 0.15^{\mathrm{a}}$ \\
Lp60 & $3.61 \pm 0.18^{\mathrm{a}}$ & $6.49 \pm 0.29^{\mathrm{a}}$ & $2.88 \pm 0.11^{\mathrm{a}}$ \\
Lp90 & $3.69 \pm 0.25^{\mathrm{a}}$ & $6.58 \pm 0.42^{\mathrm{a}}$ & $2.89 \pm 0.17^{\mathrm{a}}$ \\
\hline
\end{tabular}

${ }^{a}$ Data in the same column followed by the same lowercase letter are not significantly different $(P>0.05)$ from each other with respect to bile tolerance.

${ }^{1}$ Lp0 $=$ original strain; Lp30 = strains subcultured 30 times; Lp60 = strains subcultured 60 times; Lp90 = strains subcultured 90 times. ${ }^{2} \mathrm{OD}_{600}=$ optical density at $600 \mathrm{~nm}$.

${ }^{3} \mathrm{LT}=$ lag time.

${ }^{4} \mathrm{MRS}=$ de Man, Rogosa, and Sharpe. 
Table 4. Cell hydrophobicity and self-aggregation ability of Lactobacillus plantarum ATCC 14917 during continuous subculture1 (values are average $\pm \mathrm{SD}$ )

\begin{tabular}{|c|c|c|c|c|}
\hline Solvent or time & Lp0 & Lp30 & Lp60 & Lp90 \\
\hline \multicolumn{5}{|c|}{ Hydrophobicity (\%) } \\
\hline Chloroform & $95.65 \pm 1.12^{\mathrm{A}, \mathrm{a}}$ & $95.93 \pm 1.28^{\mathrm{A}, \mathrm{a}}$ & $96.18 \pm 1.51^{\mathrm{A}, \mathrm{a}}$ & $95.74 \pm 1.80^{\mathrm{A}, \mathrm{a}}$ \\
\hline Ethyl acetate & $74.56 \pm 2.83^{\mathrm{C}, \mathrm{a}}$ & $74.88 \pm 1.02^{\mathrm{C}, \mathrm{a}}$ & $75.01 \pm 1.92^{\mathrm{C}, \mathrm{a}}$ & $74.74 \pm 0.85^{\mathrm{C}, \mathrm{a}}$ \\
\hline \multicolumn{5}{|c|}{ Self-aggregation (\%) } \\
\hline $2 \mathrm{~h}$ & $7.81 \pm 0.52^{\mathrm{A}, \mathrm{a}}$ & $7.89 \pm 0.75^{\mathrm{A}, \mathrm{a}}$ & $8.09 \pm 1.12^{\mathrm{A}, \mathrm{a}}$ & $8.00 \pm 0.30^{\mathrm{A}, \mathrm{a}}$ \\
\hline $16 \mathrm{~h}$ & $48.88 \pm 2.36^{\mathrm{B}, \mathrm{a}}$ & $48.92 \pm 1.05^{\mathrm{B}, \mathrm{a}}$ & $49.01 \pm 1.93^{\mathrm{B}, \mathrm{a}}$ & $48.87 \pm 1.22^{\mathrm{B}, \mathrm{a}}$ \\
\hline
\end{tabular}

${ }_{\mathrm{a} ; \mathrm{A}-\mathrm{D}}$ The identical lowercase letters indicate that no significant differences were observed among different strains in cell hydrophobicity at the same solvents and no significant differences in self-aggregation ability at the same time points. Different uppercase letters indicate that significant differences $(P<0.05)$ were observed in cell hydrophobicity at different solvents and significant differences $(P<0.05)$ in self-aggregation ability at different times when tested in the same strain.

${ }^{1}$ Lp0 $=$ original strain; Lp30 = strains subcultured 30 times; Lp60 = strains subcultured 60 times; Lp90 = strains subcultured 90 times.

tabolism of L. plantarum ATCC 14917. However, the deficiency of $L a c Z / L a c L M$ genes prevents synthesis of $\beta$-galactosidase, a key enzyme for lactose metabolism, so that lactose cannot be fully used by L. plantarum ATCC 14917.

To reach and survive in the human gut is essential if probiotics are to have a beneficial effect. For this reason, acid and bile tolerance capabilities are primary screening characteristics when selecting probiotic strains (Tuomola et al., 2001). However, no sufficient studies on the stability of acid tolerance during continuous subculture were reported. Although we found the survival rate of the Lp30, Lp60, and Lp90 cultures in the presence of acid was lower than in the absence of acid, acid tolerance ability of L. plantarum still presented higher when compared with the results from previous research (Guo et al., 2017), and they would survive stomach acid and remain viable into the intestine. No significant difference was observed in acid tolerance among the Lp0, Lp30, Lp60, and Lp90 cultures, illustrating that acid tolerance was stable during continuous subculture. The LT of $L$. plantarum was short, indicating that bile tolerance was high (Kaushik et al., 2009). Because bile can destroy cell structure, it has specific side effects on living cells. However, in continuous subculture bile tolerance remained stable, signifying that the bacteria

Table 5. Pathogen inhibition activity of Lactobacillus plantarum ATCC 14917 during continuous subculture ${ }^{1,2}$

\begin{tabular}{lcccc}
\hline Indicator strain & Lp0 & Lp30 & Lp60 & Lp90 \\
\hline Staphylococcus aureus & ++ & ++ & ++ & ++ \\
Escherichia coli & + & + & + & + \\
\hline
\end{tabular}

${ }^{1} \mathrm{Lp} 0=$ original strain; Lp30 $=$ strains subcultured 30 times; Lp60 $=$ strains subcultured 60 times; Lp90 = strains subcultured 90 times.

${ }^{2}+=$ inhibition zone diameter 5 to $10 \mathrm{~mm} ;++=$ inhibition zone diameter 10 to $15 \mathrm{~mm}$. could still endure high bile salt and survive in the intestine.

Probiotics form a stable biological barrier by adhering to the intestinal mucosa, and have important physiological functions such as inhibiting the invasion and proliferation of pathogenic bacteria. Perez et al. (1998) studied the relationships among adhesion, cell hydrophobicity, and self-aggregation ability of Bifidobacterium species finding that adhesion was strongly correlated with cell hydrophobicity and self-aggregation ability; isolates with strong cell hydrophobicity and

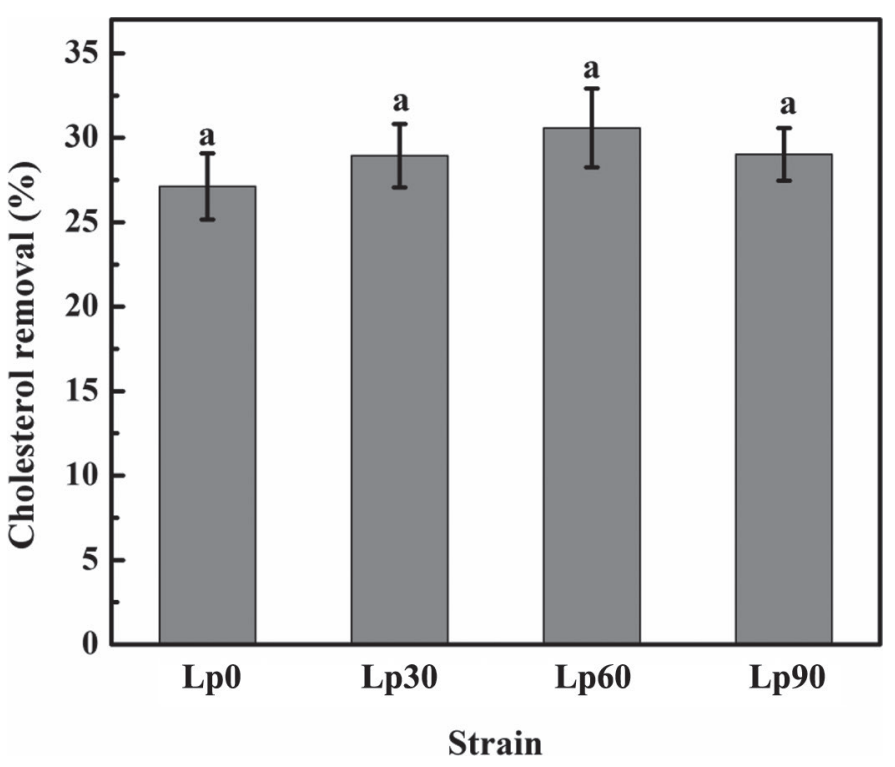

Figure 2. Cholesterol removal ability of Lactobacillus plantarum ATCC 14917 during continuous subculture. Lp0 = original strain; Lp30 $=$ strains subcultured 30 times; Lp60 $=$ strains subcultured 60 times; Lp90 = strains subcultured 90 times. Error bars indicate SD. The identical lowercase letters indicate that no significant differences were observed in the cholesterol removal among Lp0, Lp30, Lp60, and Lp90 strains. 
Table 6. Mapping of the tested sequences to the reference sequence of Lactobacillus plantarum ATCC 14917

\begin{tabular}{lccc}
\hline Sample $^{1}$ & Count $^{2}$ & $\begin{array}{c}\text { Number } \\
\text { of base }^{3}\end{array}$ & $\begin{array}{c}\text { Match } \\
\text { rate (\%) }\end{array}$ \\
\hline Lp0-1 & 111,024 & $5,329,152$ & 98.35 \\
Lp0-2 & 111,941 & $5,373,168$ & 98.52 \\
Lp30-1 & 138,605 & $6,653,040$ & 98.66 \\
Lp30-2 & 128,236 & $6,155,328$ & 98.52 \\
Lp60-1 & 120,684 & $5,792,832$ & 98.55 \\
Lp60-2 & 121,142 & $5,814,816$ & 98.84 \\
Lp90-1 & 109,438 & $5,253,024$ & 98.55 \\
Lp90-2 & 115,582 & $5,547,936$ & 98.54 \\
\hline
\end{tabular}

${ }^{1} \mathrm{Lp} 0=$ original strain; Lp30 = strains subcultured 30 times; Lp60 = strains subcultured 60 times; Lp90 = strains subcultured 90 times.

${ }^{2}$ The count of mapped reads.

${ }^{3}$ The number of base for mapped reads.

self-aggregation ability had higher adhesion ability. Bellon-Fontaine et al. (1996) found the adhesion in alkaline solvent was lower in acidic solvents, indicating that bacteria are electron donors and weak electron acceptors. This is consistent with our results: adhesion in the acidic solvent chloroform was higher than in alkaline solvent ethyl acetate. In addition, the adhesion of $L$. plantarum in xylene solvent we detected was sig- nificantly higher than the result of Yadav et al. (2016). Analysis of the cell hydrophobicity and self-aggregation ability of Lp0, Lp30, Lp60, and Lp90 cultures showed that they had strong adhesion characteristics, which is similar to the previous study (Yadav et al., 2016), and they were maintained during continuous subculture. Elo et al. (1991) studied Lactobacillus rhamnosus GG showing that adhesion characteristics decreased significantly after storage for $3.5 \mathrm{yr}$ in MRS broth. This variability shows that adhesion stability should be evaluated routinely during long-term industrial processes such as continuous subculturing and long-term storage.

Lactobacillus plantarum was shown to effectively inhibit the growth of $S$. aureus and $E$. coli by producing $\mathrm{H}_{2} \mathrm{O}_{2}$, diacetyl, bacteriocin, and other antimicrobial substances (Niku-Paavola et al., 1999), thereby maintaining the intestinal flora balance in humans and animals. Lash et al. (2005) found that the cell-free supernatant of L. plantarum could inhibit the growth of most grampositive bacteria (e.g., S. aureus) and gram-negative bacteria (e.g., E. coli), which was consistent with our results. Comprehensive analysis of the acid and bile tolerance results described above indicated that $L$. plantarum can employ its antibacterial properties after
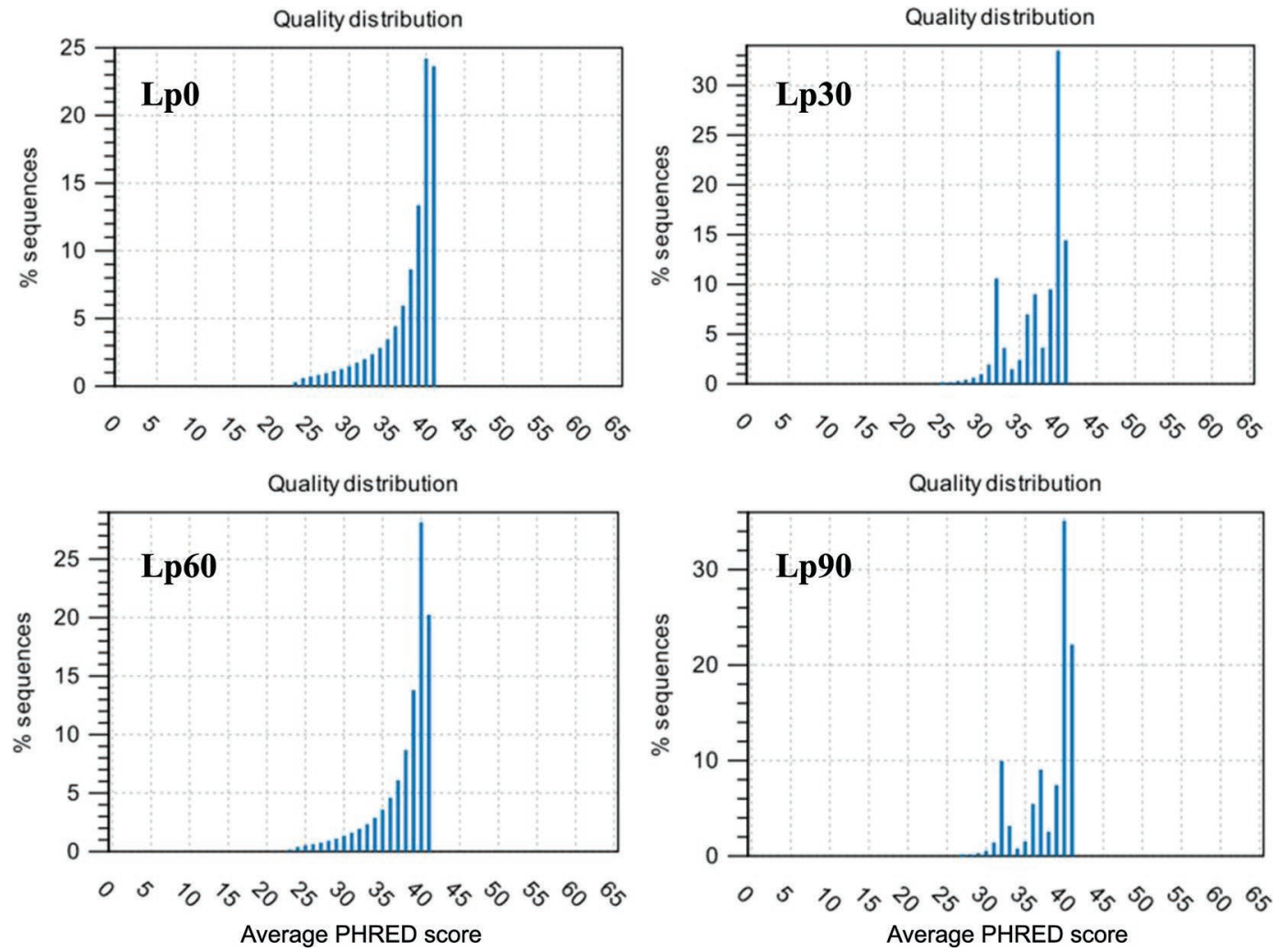

Figure 3. The distribution of average sequence quality scores. x-axis: PHRED score; y-axis: number of sequences observed at that quality score normalized to the total number of sequences. Lp0 = original strain; Lp30 = strains subcultured 30 times; Lp60 = strains subcultured 60 times; Lp90 = strains subcultured 90 times. 


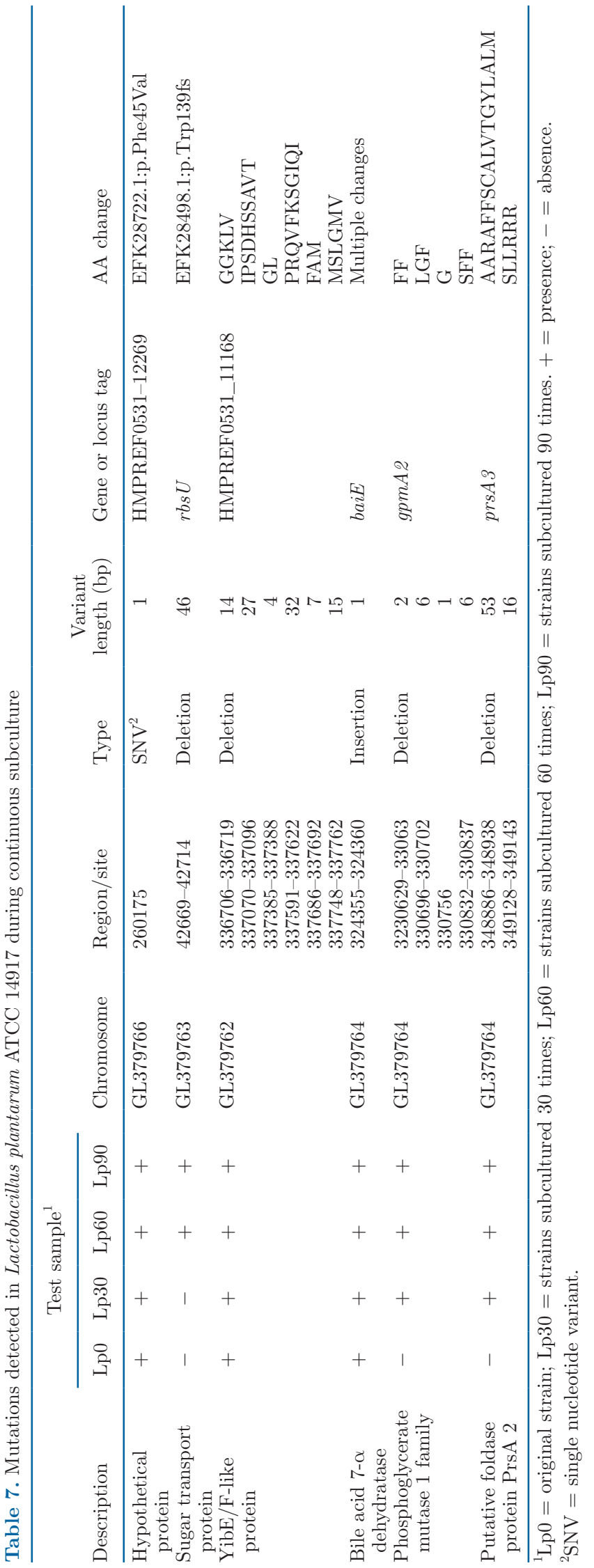

passing through the human gastrointestinal tract in a certain amount and activity, and this characteristic was stable throughout the continuous subculture.

Cholesterol is an indispensable substance for human cells, whereas high cholesterol levels are often considered as a risk for heart disease (Guo et al., 2001). Lactobacillus plantarum could lower the serum cholesterol levels. In present study, we demonstrated that $L$. plantarum ATCC 14917 had a stable ability to remove cholesterol in the culture media during continuous subculture, and was higher than the average cholesterol removal rate $(19.4 \%)$ of L. plantarum presented in Belviso et al. (2009).

With the exception of carbohydrate metabolism, phenotypic characteristics of $L$. plantarum ATCC 14917 were stable during continuous subculture in MRS broth. The reason for changes in carbohydrate metabolism may be due to mutations in the gene related to the regulation of phosphoglycerate mutase 1 family; this enzyme is critical in glycolysis and gluconeogenesis and associated with microbial carbohydrate utilization (Ashton et al., 2001). The phosphoglycerate mutase 1 family was only present in the Lp30, Lp60, and Lp90 cultures, which may explain why the ability to use Dsorbitol, $\alpha$-methyl-D-mannose, D-raffinose, gentiobiose, and gluconate had changed in Lp30, Lp60, and Lp90 cultures compared with Lp0 cultures. Sugar transport protein is a family of bacterial sugar transporters approximately 300 residues long including glucose uptake proteins, ribose transport proteins, and putative and hypothetical membrane proteins, which are potentially involved in sugar transport across bacterial membranes (Fiegler et al., 1999). In this study, the gene mutations conferring sugar transport protein were only detected in Lp60 and Lp90 cultures, whereas the carbohydrate metabolism patterns of Lp30, Lp60, and Lp90 were the same, suggesting the mutation or the gene may not involve regulation of the carbohydrate metabolism. The mutation related to bile acid $7-\alpha$ dehydratase participates in the 7-dehydroxylation process associated with bile acid degradation, which allows conversion of primary bile acids into secondary bile acids and is important for ensuring the steady state of bile acid metabolism and maintaining bile acid tolerance (Nicolas et al., 2016). Most members of the YibE/F-like protein family are annotated as putative membrane proteins, and the sequence contains a high proportion of hydrophobic residues (NCBI, 2015), which may help Lp0, Lp30, Lp60, and Lp90 cultures maintain a high level of cell hydrophobicity. Mutation of the gene encoding putative foldase protein PrsA 2 could alter cell wall or membrane protein profiles as well as cell surface related phenotypes, including self-aggregation and cell hydrophobicity. Putative foldase protein PrsA 2 deficient 


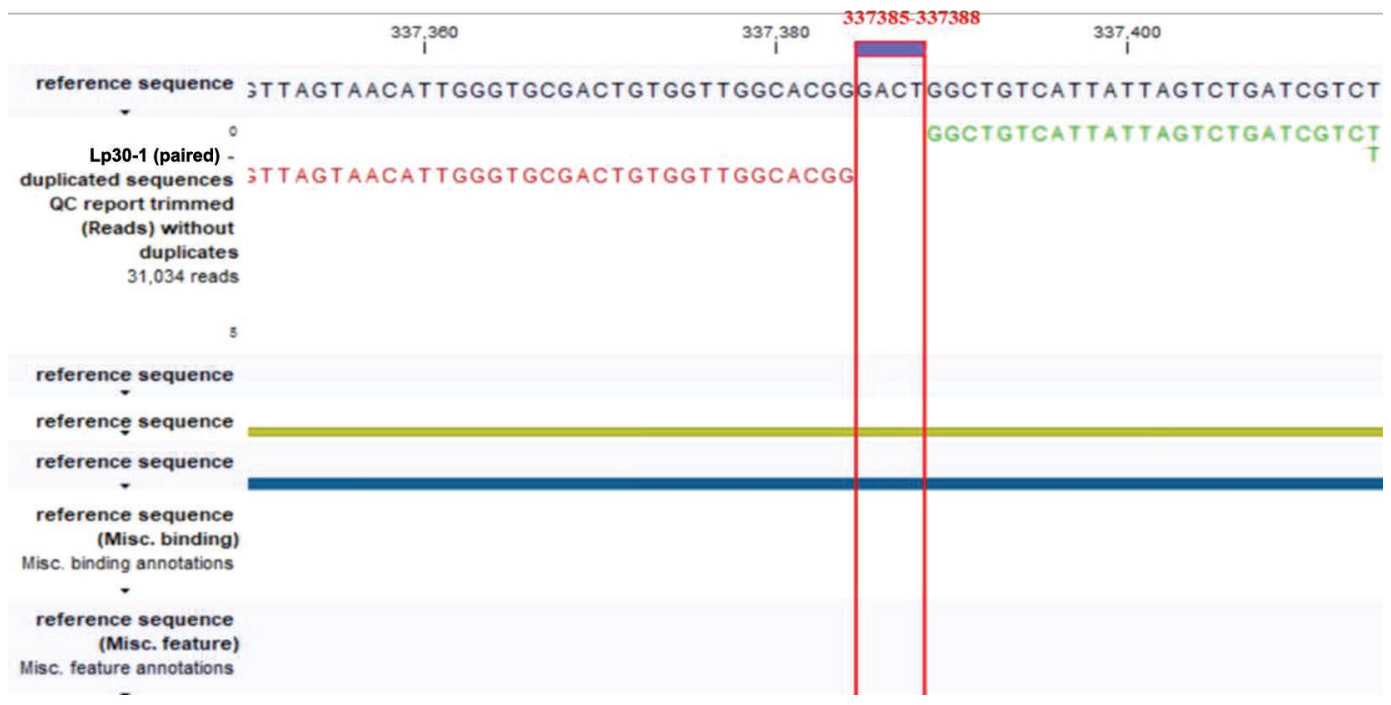

Figure 4. The track list of reference sequence and the sequence of 30-d culture of Lactobacillus plantarum ATCC 14917. Misc. $=$ miscellaneous; QC = quality control; Lp30 = strains subcultured 30 times.

cultures show higher self-aggregation ability and cell hydrophobicity (Guo et al., 2013). In our study, no significant difference $(P>0.05)$ in self-aggregation ability and cell hydrophobicity during continuous subculture was recorded.

\section{CONCLUSIONS}

Overall, by comparing the phenotypic and genotypic characteristics of $L$. plantarum ATCC 14917 cultures during continuous subculture, we found that the metabolism of D-sorbitol, $\alpha$-methyl-D-mannose, D-raffinose, gluconate, and gentiobiose were changed. The deficiency of LacZ/LacLM genes leads to the failure for L. plantarum ATCC 14917 to ferment the milk. Nevertheless, the probiotic traits of L. plantarum ATCC 14917 remained stable throughout the continuous subculture, indicating L. plantarum ATCC 14917 is a stable probiotic strain that can be subcultured and used in long-term food industry production. The present study provides a way to evaluate the stability of a probiotic strain.

\section{ACKNOWLEDGMENTS}

This research was supported by the China Scholarship Council (file no. 201806875010), the National Natural Science Foundation of China (31771973), the Major Project for Transformation of Scientific and Technological Achievements of Shaanxi Province (2016KTCG0112, China), and the Fundamental Research Funds for the Central Universities (GK201703063, GK201806008).

\section{REFERENCES}

Agostoni, C., I. Axelsson, C. Braegger, O. Goulet, B. Koletzko, K. F. Michaelsen, J. Rigo, R. Shamir, H. Szajewska, D. Turck, L. T. Weaver, and ESPGHAN Committee on Nutrition. 2004. Probiotic bacteria in dietetic products for infants: A commentary by the ESPGHAN Committee on Nutrition. J. Pediatr. Gastroenterol. Nutr. 38:365-374.

AOAC International. 1999. Official Methods of Analysis. 16th ed. 5th rev. AOAC International, Gaithersburg, MD.

Ashton, P. D., R. S. Curwen, and R. A. Wilson. 2001. Linking proteome and genome: How to identify parasite proteins. Trends Parasitol. 17:198-202.

Bachmann, H., M. Starrenburg, D. Molenaar, and M. Kleerebezem. 2012. Microbial domestication signatures of Lactococcus lactis can be reproduced by experimental evolution. Genome Res. 22:115124.

Bellon-Fontaine, M. N., J. Rault, and C. J. V. Oss. 1996. Microbial adhesion to solvents: A novel method to determine the electrondonor/electron-acceptor or Lewis acid-base properties of microbial cells. Colloid Surface B 7:47-53.

Belviso, S., M. Giordano, P. Dolci, and G. Zeppa. 2009. In vitro cholesterol-lowering activity of Lactobacillus plantarum and Lactobacillus paracasei strains isolated from the Italian Castelmagno PDO cheese. Dairy Sci. Technol. 89:169-176.

Bryant, J., C. Chewapreecha, and S. D. Bentley. 2012. Developing insights into the mechanisms of evolution of bacterial pathogens from whole-genome sequences. Future Microbiol. 7:1283-1296.

Buckling, A., R. Craig Maclean, M. A. Brockhurst, and C. Nick. 2009. The Beagle in a bottle. Nature 457:824-829.

Collado, M. C., J. Meriluoto, and S. Salminen. 2008. Adhesion and aggregation properties of probiotic and pathogen strains. Eur. Food Res. Technol. 226:1065-1073.

Dong, Y., F. Zhang, B. Wang, J. Gao, J. Zhang, and Y. Shao. 2019. Laboratory evolution assays and whole-genome sequencing for the development and safety evaluation of Lactobacillus plantarum with stable resistance to gentamicin. Front. Microbiol. 10:1235.

Dunne, C., L. O'Mahony, L. Murphy, G. Thornton, D. Morrissey, S. O'Halloran, and C. Daly. 2001. In vitro selection criteria for probiotic bacteria of human origin: Correlation with in vivo findings. Am. J. Clin. Nutr. 73:386S

Elo, S., M. Saxelin, and S. Salminen. 1991. Attachment of Lactobacillus casei strain GG to human colon carcinoma cell line Caco- 
2: Comparison with other dairy strains. Lett. Appl. Microbiol. $13: 154-156$.

FAO and WHO. 2006. Probiotic in foods. Health and nutritional properties and guidelines for evaluation. FAO Food Nutr. Paper 85.

Fiegler, H., J. Bassias, I. Jankovic, and R. Bruckner. 1999. Identification of a gene in Staphylococcus xylosus encoding a novel glucose uptake protein. J. Bacteriol. 181:4929.

Gilliland, S., C. Nelson, and C. Maxwell. 1985. Assimilation of cholesterol by Lactobacillus acidophilus. Appl. Environ. Microbiol. 49:377-381.

Guo, C., and L. Zhang. 2010. Cholesterol-lowering effects of probiotics-A review. Wei Sheng Wu Xue Bao 50:1590-1599.

Guo, L., T. Wu, W. Hu, X. He, S. Sharma, P. Webster, and W. Shi. 2013. Phenotypic characterization of the foldase homologue PrsA in Streptococcus mutans. Mol. Oral Microbiol. 28:154-165.

Guo, L., L. Yang, and G. Huo. 2001. Cholesterol removal by Lactobacillus plantarum isolated from homemade fermented cream in Inner Mongolia of China. Czech J. Food Sci. 29:219-225.

Guo, Y., X. Tian, R. Huang, X. Tao, N. Shah, H. Wei, and C. Wan. 2017. A physiological comparative study of acid tolerance of Lactobacillus plantarum ZDY 2013 and L. plantarum ATCC 8014 at membrane and cytoplasm levels. Ann. Microbiol. 67:669-677.

He, S., C. Ran, C. Qin, S. Li, H. Zhang, W. M. de Vos, E. Ring, and Z. Zhou. 2017. Anti-infective effect of adhesive probiotic Lactobacillus in fish is correlated with their spatial distribution in the intestinal tissue. Sci. Rep. 7:13195.

Hyronimus, B., C. L. Marrec, A. H. Sassi, and A. Deschamps. 2000 Acid and bile tolerance of spore-forming lactic acid bacteria. Int. J. Food Microbiol. 61:193-197.

Kaushik, J. K., K. Ashutosh, R. K. Duary, A. K. Mohanty, G. Sunita, and V. K. Batish. 2009. Functional and probiotic attributes of an indigenous isolate of Lactobacillus plantarum. PLoS One 4:e8099.

Kerr, J. 2004. Manual of Clinical Microbiology, 8th ed. J. Clin. Pathol. 47:111.

Lash, B. W., T. H. Mysliwiec, and H. Gourama. 2005. Detection and partial characterization of a broad-range bacteriocin produced by Lactobacillus plantarum (ATCC 8014). Food Microbiol. 22:199204.

Lin, W. H., B. Yu, S. H. Jang, and H. Y. Tsen. 2007. Different probiotic properties for Lactobacillus fermentum strains isolated from swine and poultry. Anaerobe 13:107-113.

Lu, M., Y. Tang, M. Li, P. Yang, Z. Liu, J. Yuan, and P. Zheng. 2015. Co-administration of cholesterol-lowering probiotics and anthraquinone from Cassia obtusifolia L. ameliorate non-alcoholic fatty liver. PLoS One 10:e0138078.

Manalis, N., and F. Magkanari. 2006. Production of traditional Greek yoghurt using Lactobacillus strains with probiotic potential as starter adjuncts. Int. Dairy J. 16:52-60.

Medina, R., M. Katz, S. Gonzalez, and G. Oliver. 2001. Characterization of the lactic acid bacteria in ewe's milk and cheese from northwest Argentina. J. Food Prot. 64:559.

Milesi, M. M., P. L. H. McSweeney, and E. R. Hynes. 2008. Viability and contribution to proteolysis of an adjunct culture of Lactobacil- lus plantarum in two model cheese systems: Cheddar cheese-type and soft-cheese type. J. Appl. Microbiol. 105:884-892.

Morelli, L., and E. Campominosi. 2002. Genetic stability of Lactobacillus paracasei ssp. paracasei F 19. Microb. Ecol. Health Dis. 14:14-16.

NCBI (National Center for Biotechnology Information). 2015. CDD Conserved Protein Domain Family: YibE-F. Accessed Nov. 19, 2015. https://www.ncbi.nlm.nih.gov/Structure/cdd/cddsrv.cgi ?uid $=311730$.

Nicolas, S., D. Lyne, B. Markus, B. Sanrine, M. Terrazos, M. Laure, C. Schürch, K. McCoy, S. Kuehne, N. Minton, B. Stecher, R. BernierLatmani, and S. Hapfelmeier. 2016. Functional intestinal bile acid $7 \alpha$-dehydroxylation by Clostridium scindens associated with protection from Clostridium difficile infection in a gnotobiotic mouse model. Front. Cell Infect. Microbiol. 6:1-15.

Nieto-Arribas, P., J. M. Poveda, S. Seseña, L. Palop, and L. Cabezas. 2009. Technological characterization of Lactobacillus isolates from traditional Manchego cheese for potential use as adjunct starter cultures. Food Control 20:1092-1098.

Niku-Paavola, M., A. Laitila, T. Mattila-Sandholm, and A. Haikara. 1999. New types of antimicrobial compounds produced by Lactobacillus plantarum. J. Appl. Microbiol. 86:29-35.

Parvez, S., K. Malik, S. Kang, and H. Kim. 2006. Probiotics and their fermented food products are beneficial for health. J. Appl. Microbiol. 100:1171-1185.

Patel, A. K., J. J. Ahire, S. P. Pawar, B. L. Chaudhari, and S. B. Chincholkar. 2009. Comparative accounts of probiotic characteristics of Bacillus spp. isolated from food wastes. Food Res. Int. 42:505-510.

Perez, P. F., Y. Minnaard, E. A. Disalvo, and G. L. De Antoni. 1998 Surface properties of bifidobacterial strains of human origin. Appl. Environ. Microbiol. 64:21-26.

Tomaro-Duchesneau, C., M. L. Jones, D. Shah, P. Jain, S. Saha, and S. Prakash. 2014. Cholesterol assimilation by Lactobacillus probiotic bacteria: An in vitro investigation. BioMed Res. Int. 2014:380316.

Tuomola, E., R. Crittenden, M. Playne, E. Isolauri, and S. Salminen. 2001. Quality assurance criteria for probiotic bacteria. Am. J. Clin. Nutr. 73:393S.

Walker, D. K., and S. E. Gilliland. 1993. Relationship among bile tolerance, bile salt deconjugation, and assimilation of cholesterol by Lactobacillus acidophilus. J. Dairy Sci. 76:956-961.

Wang, P., Z. Wu, J. Wu, D. Pan, X. Zeng, and K. Cheng. 2016. Effects of salt stress on carbohydrate metabolism of Lactobacillus plantarum ATCC 14917. Curr. Microbiol. 73:491-497.

Yadav, R., A. K. Puniya, and S. Pratyoosh. 2016. Probiotic properties of Lactobacillus plantarum RYPR1 from an indigenous fermented beverage Raabadi. Front. Microbiol. 7:1683.

\section{ORCIDS}

Yuyu Shao 이 https://orcid.org/0000-0002-1826-8203 